\title{
Experimental validation as an integral component of computational fluid dynamics research
}
G. Doig ${ }^{1}$
T. Barber ${ }^{2}$
A. Neely ${ }^{3}$
D. $\mathrm{Myre}^{4}$

(Received 31 January 2010; revised 21 April 2010)

\begin{abstract}
As experimental fluid dynamics becomes increasingly expensive and time consuming, researchers become more reliant on computational fluid dynamics (CFD) solutions. However for many applications, the use of CFD alone is not enough to provide confidence. Using the example of compressible flow ground effect aerodynamics, the use of an integrated computational/experimental fluid dynamics research program is described. Relatively cheap and simple experiments are designed using CFD to provide suitable and thorough validation data for more complex CFD flows. Extra advantages of the implementation of both techniques by the same team of researchers are that errors are minimised, and issues of miscommunication between two disparate groups of numerical and experimental researchers disappear.
\end{abstract}

http://anziamj . austms.org.au/ojs/index.php/ANZIAMJ/article/view/2617 gives this article, (c) Austral. Mathematical Soc. 2010. Published May 31, 2010. ISSN 1446-8735. (Print two pages per sheet of paper.) 


\section{Contents}

1 Introduction

C266

2 Compressible ground effect aerodynamics

C268

3 Methodology

C269

4 Conclusions

C276

References

C277

\section{Introduction}

Computational fluid dynamics (CFD), particularly as applied in industry, is becoming increasingly divorced from experimental fluid dynamic (EFD) programs which are traditionally more expensive and time consuming. CFD users are therefore becoming increasingly reliant on validation data which either predates the modern era of high performance computing, is inadequately described in literature, or is removed from the actual problem being researched numerically. To use an example related directly to the work discussed in this paper, the AGARD report Experimental Data Base for Computer Program Assessment [1] of 1979, the benchmark for transonic aerofoil CFD validation since its publication, exhibits all of the above issues when used in the present context. Although extensive, the results are not of similar resolution to that which can be obtained from CFD, the three dimensionality of the flowfield is not discussed in detail, and neither are the slotted walls of the test section which are not easy to replicate in CFD in any case. These factors led researchers in recent times to performing a kind of forensic CFD to establish what the full imperfect flowfield would have been [2].

This article discusses the intrinsic value of the design and execution of simple experimental fluid dynamic programs as a dedicated validation tool for 


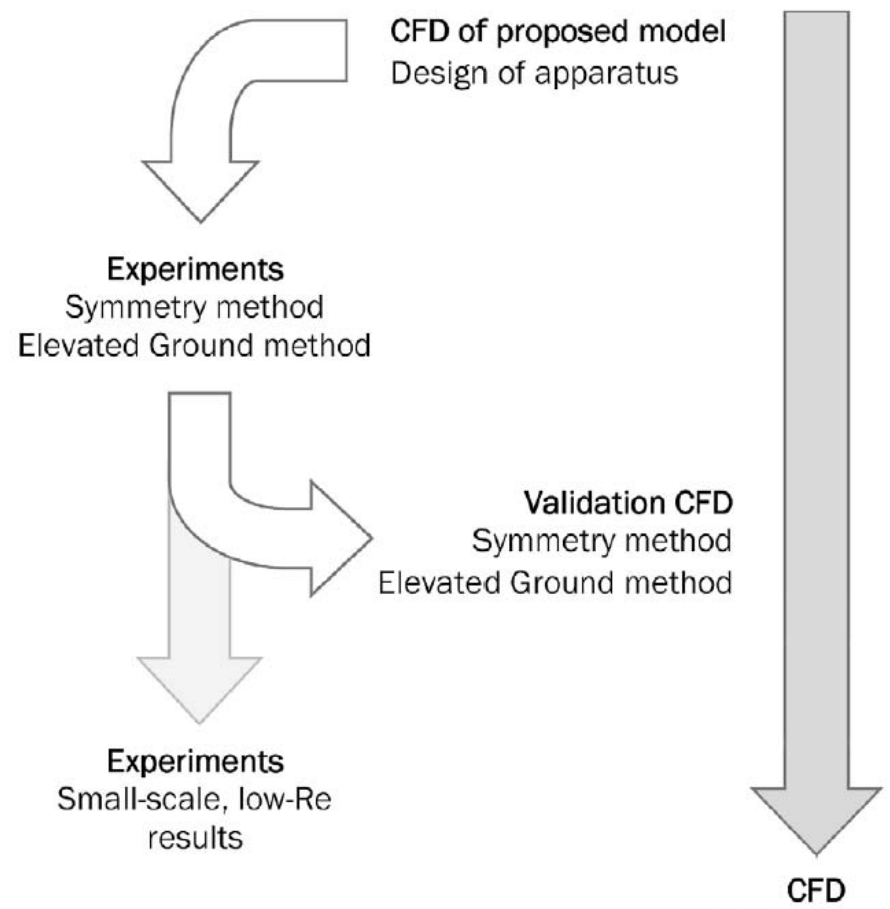

Full Scale, high-Re results

FiguRE 1: Role of transonic experiments in cooperation with CFD. 
numerical modelling, both in terms of data for comparison and ancillary insight into the problem being investigated. This shifting of emphasis, whereby experiments support CFD rather than vice versa, reflects the role reversal currently ingraining itself in fluid dynamic research and development programs in many fields. This is a particular issue in aerodynamics, where the value of the wind tunnel is increasingly debated.

The example of compressible flow ground effect aerodynamics is used to illustrate that relatively cheap and simple experiments can be designed to provide suitable and thorough validation data for complex flows. As there is no practical or cost effective way to study full scale transonic and supersonic ground effect flows with conventional experimental techniques, CFD becomes an attractive tool in this instance. Precisely because of the lack of simple means for experimental testing, it is tempting to become reliant on CFD alone. However, dependence on CFD by itself to investigate transonic and supersonic flowfields, particularly with regards to ground interactions, does not engender a sufficient level of confidence in the results

\section{Compressible ground effect aerodynamics}

Ground effect aerodynamics have mostly been studied in a subsonic context $[3,4]$, but there are also transonic and supersonic applications in which the proximity of the ground is an important parameter, such as land speed record cars, low flying military aircraft, or projectiles close to the ground or solid obstacles. In order to conduct experiments to study the aerodynamics of supersonic objects in ground effect, particular consideration must be given to the way in which the ground is represented. In a wind tunnel environment with a fixed model, ideally a moving ground would be used for the greatest physical realism $[4,5]$, but this is impractical at supersonic speeds. It is arguably more straightforward to move the object through quiescent air using, for example, a rocket sled testing facility [5] or a ballistic range [6], but in addition to the enhanced complexity of all diagnostics related to free 
flight measurements, these facilities are generally expensive and may also be subject to restricted military access.

By contrast, supersonic blowdown tunnels are relatively common and accessible, and thus this study is an investigation of the applicability of the most suitable subsonic ground effect modelling techniques for supersonic applications in such facilities. The extension to transonic conditions is then examined.

A much improved understanding of the aerodynamic influence of compressible ground effects and of shock/ground interaction would benefit research into high speed subsonic (freestream Mach number, $M=0.4$ ) wing-inground-effect aircraft [7], as well as magnetic levitation space vehicle launch systems [8] and rail vehicles [9].

\section{$3 \quad$ Methodology}

In this study wind tunnel experiments with various model arrangements, using pressure tappings and schlieren photography, were carried out and supplemented by numerical simulations of the experiments in order to better quantify the relative merits of each method. The research described involves a constructive relationship between experimental testing and Reynolds Averaged Navier Stokes (RANS) CFD; in effect using the CFD to confirm the effectiveness of the experiments and using the experiments to validate the computational approach to the point where CFD used alone furthers understanding of the flows investigated.

Further details of the experimental design, setup and analysis are reported elsewhere $[10,11]$. Similarly, Doig [10] described in detail the lengthy validation and verification process undertaken before any CFD results were used. The emphasis here is on the use of the two methods to produce an integrated solution; specific details of each method are omitted here for brevity and clarity. 


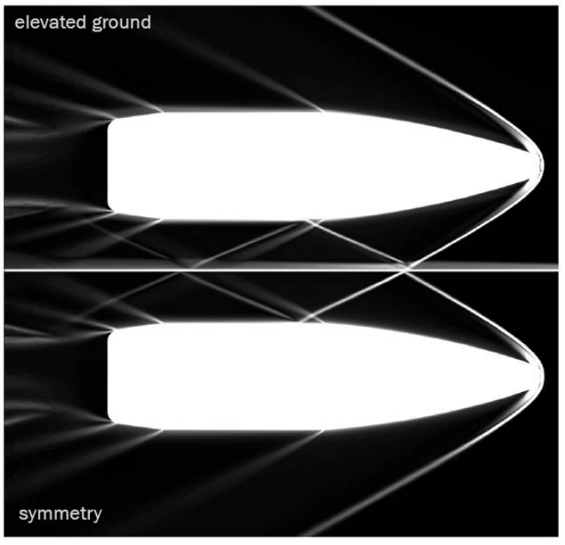

(a) symmetry and elevated ground methods.

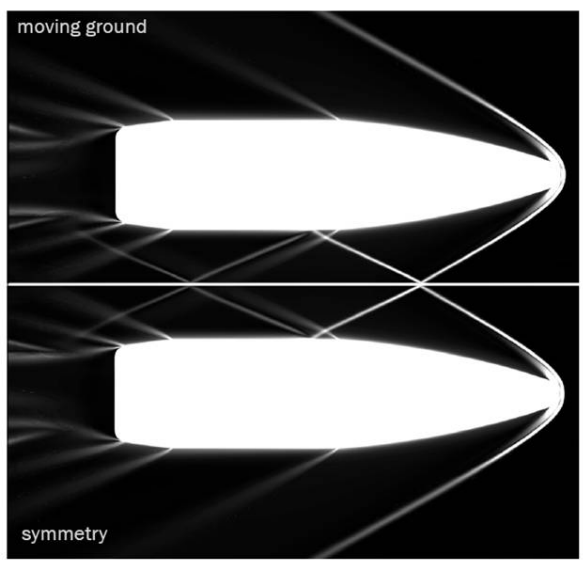

(b) symmetry method and a moving ground.

FiguRE 2: CFD density contour comparisons at $h / c=0.5$.

The two methods for ground simulation examined in more detail here are the elevated ground plane, which reduces the extent of the ground boundary layer, and a symmetry ground condition, which in the experimental case requires two identical 'mirror image' models. Wind tunnel experiments and numerical simulations better quantify the relative merits of each method.

The CFD modelling was an integral part of this process, as the creation of a moving ground in CFD is a trivial task. The two less ideal experimental options were then able to be compared to the best case scenario.

CFD was used in the initial design of the experiments to identify any potential problems with the apparatus, such as wall or sting interference, and to approximate the forces acting on the models to ensure that the construction would be of sufficient strength. The numerical results also helped identify approximately the Mach numbers at which the most interesting or unusual flowfields would be produced (for instance, when the critical Mach number would be reached or when transient behavior might be observed). 


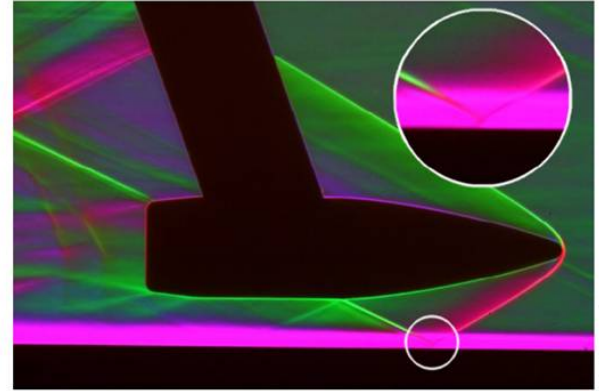

(a) elevated ground test $\mathrm{h} / \mathrm{d}=0.5$ (inset, shock/boundary layer interaction detail).

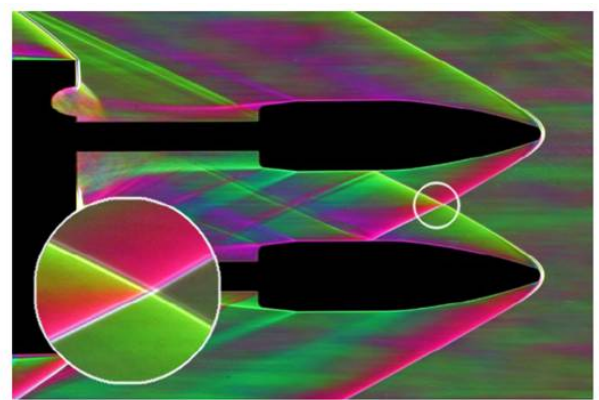

(b) symmetry method test $\mathrm{h} / \mathrm{d}=0.52$ (inset, crossing shock interaction detail).

FigURE 3: Experimental colour schlieren of modelling options.

To investigate the flow around a projectile geometry at Mach 2.4 in ground effect, the elevated ground was designed to extend far enough upstream of the projectile (approximately $140 \mathrm{~mm}$ ) such that any disturbances generated by the leading edge of the ground would not impinge directly upon the projectile. The boundary layer thickness on the ground plate at the location of the projectile nose was approximately $2.5 \mathrm{~mm}$. At low $\mathrm{h} / \mathrm{d}$ clearances, the boundary layer on the elevated ground is significant in relation to the size of the test model, as evidenced in Figure 2, in particular downstream of the impingement of the bow shock generated by the projectile model. Figure 3 indicates that at the projectile base the ground boundary layer increases to approximately $3.8 \mathrm{~mm}$, which corresponds to about $45 \%$ of the distance $h$ in this flowfield. The symmetry method produced results (in terms of pressure distribution for all cases examined) closer to that of an ideal moving ground than the elevated ground plane. We recommend the symmetry method for all conceivable wind tunnel studies of supersonic ground effect.

The presence of any ground boundary layer noticeably distorts the flow downstream of the first shock/ground interaction. However, it could be beneficial to use the elevated ground method, in particular if the ground plane were 


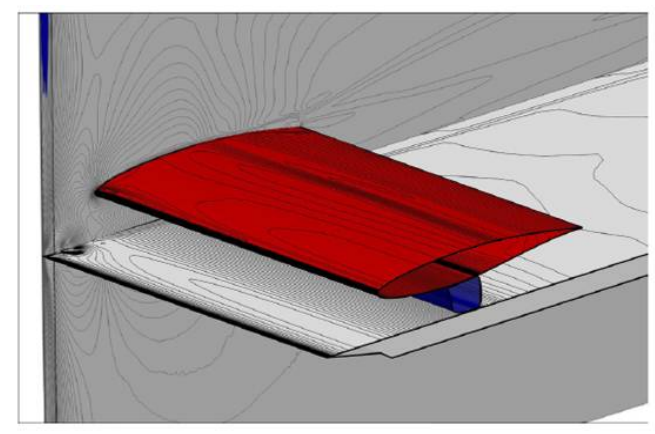

(a) contours of density and areas of supersonic flow (blue).

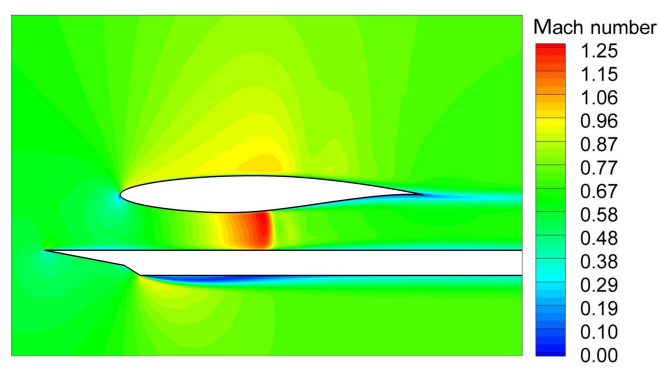

(b) contours of Mach number at the midspan symmetry plane.

FigURE 4: CFD of 2822 USNA with elevated ground plane.

extensively instrumented or thermochromic liquid crystals were used [12]. This would provide some potentially useful information about the footprint of the shocks propagating away from the model which could not be obtained with the symmetry method. There may also be cases in which the modifications of the pressure distribution caused by the boundary layer on the ground plate have a negligible net effect on the resulting aerodynamic forces, but this would have to be verified for each application.

The approach was extended to transonic ground effect, to determine if the symmetry method is also a feasible approximation for this more complex aerodynamic regime. The transonic wind tunnel of the US Naval Academy in Annapolis, Maryland was used for the transonic tests. The tunnel features a test section of cross-sectional area of $0.0418 \mathrm{~m}^{2}$, with dimensions of $0.2057 \mathrm{~m} \times 0.2032 \mathrm{~m}$. Tests were made with the porous tunnel floor and ceiling fully closed in order to facilitate more reliable CFD modelling of the test conditions with simple boundaries, as is commonly recommended (but seldom implemented) for any code validation of this nature [13]. The wing was an RAE2822 section-AGARD [1] records the coordinates - with a chord of nominally $60 \mathrm{~mm}$ and a span of $180 \mathrm{~mm}$. 


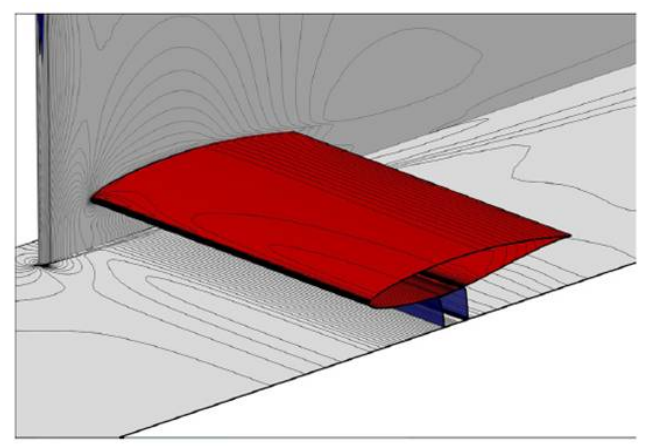

(a) contours of density and areas of supersonic flow (blue).

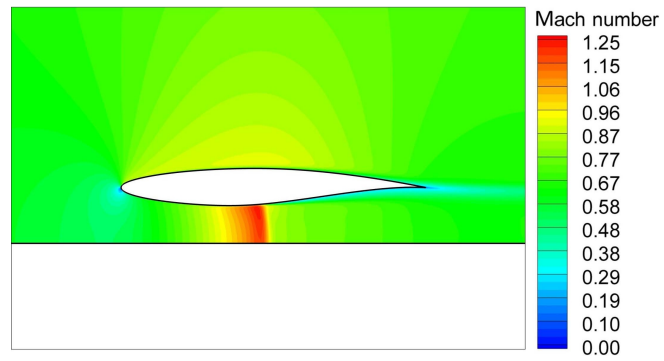

(b) contours of Mach number at the midspan symmetry plane.

FiGURE 5: CFD of 2822 USNA with symmetry ground plane.

Four cases of different high subsonic Mach number were studied; the case of $M=0.646$, with an incidence of $0^{\circ}$ and ground clearance of $h / c=0.128$ is described here. The figures show data from a plane at the mid-span of the wing where the flow was determined to be reasonably two dimensional. The lower surface shock occurs at around $x / c=0.46$, behind the point of maximum wing thickness (and thus behind the point of lowest ground clearance). In the elevated ground case, the peak upper surface Mach number is markedly increased, and the flow is near sonic. This indicates that at a freestream Mach number slightly higher than that of this particular experiment, an upper surface shock would have formed with the elevated ground where it would not have with the symmetry method, due to the additional flow displacement from the presence of the ground boundary layer. The flow is also increasingly accelerated in the gap between the wing and the ground as there is a smaller effective clearance, such that the lower surface shock in the elevated ground case is stronger than that observed with the symmetry method as illustrated in the pressure distributions at the mid-span in Figure 6 . This causes a fairly strong shock/boundary layer interaction on the elevated ground, which thickens the boundary layer downstream. 


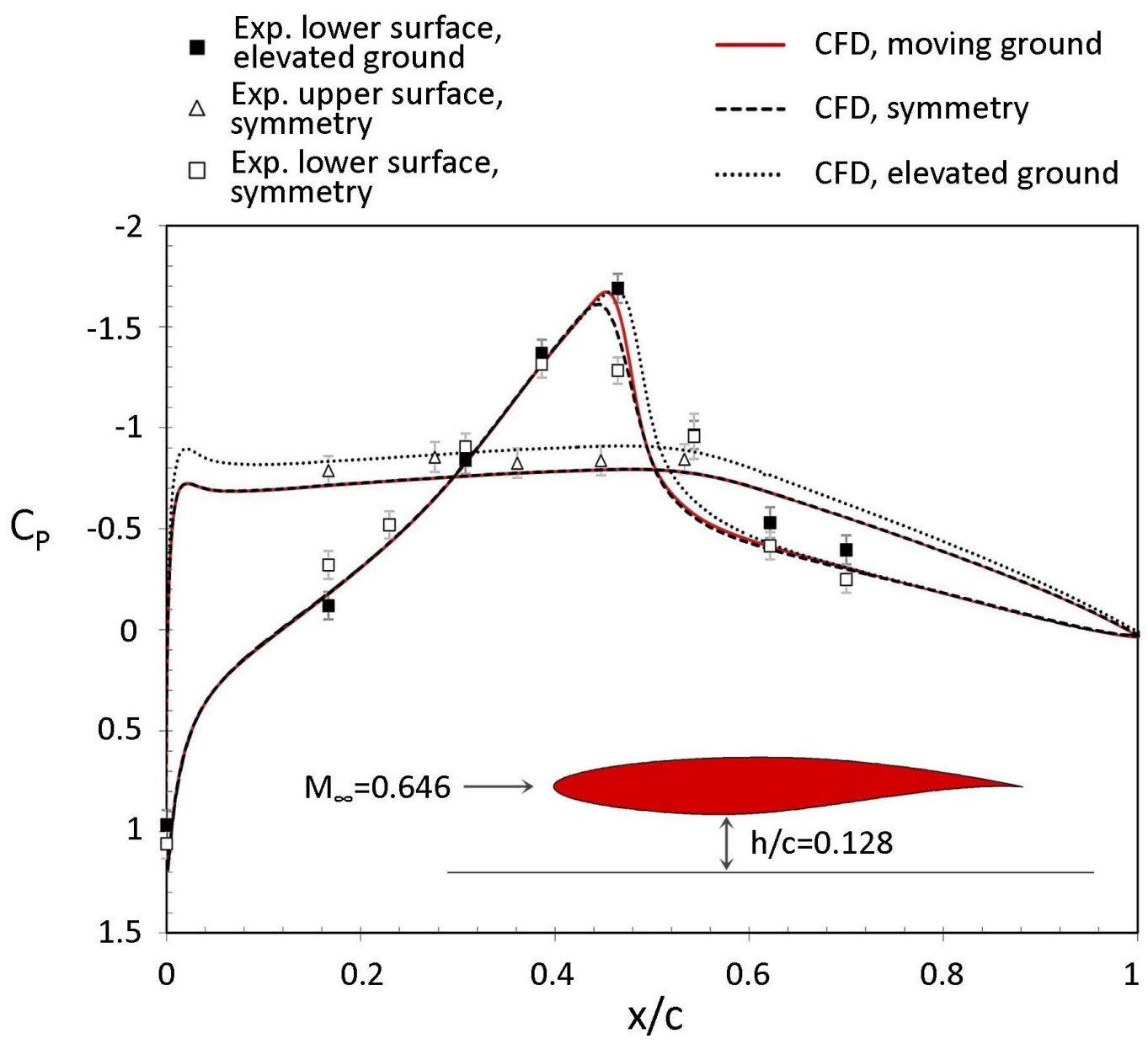

FiguRE 6: Time averaged pressure distribution, comparisons of experimental symmetry, moving ground and elevated ground methods to CFD, at the midspan plane on the wing. 


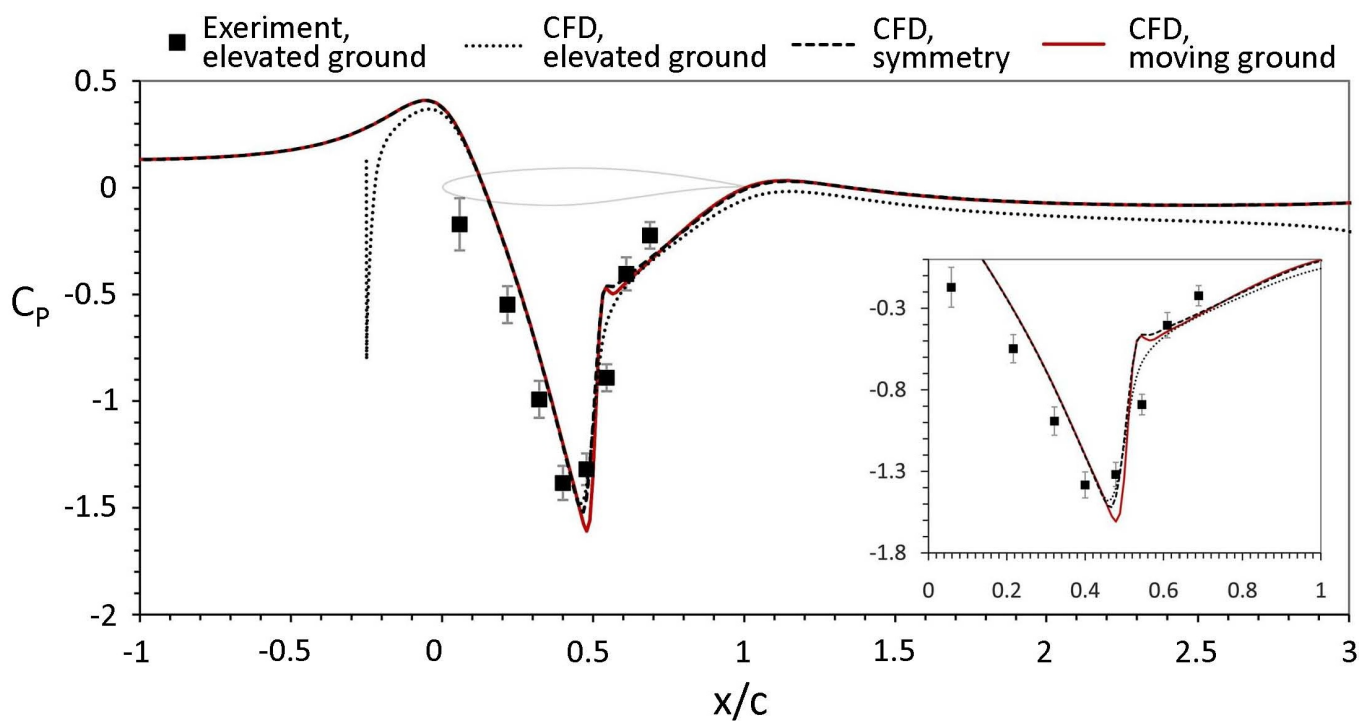

FiguRE 7: Time averaged pressure distribution, comparisons of experimental symmetry, moving ground and elevated ground methods to CFD at the midspan plane on the ground plane. 
Figure 7 presents ground plane pressure distributions to show the character of this interaction. The symmetry and elevated ground methods show a strong shock presence and accompanying pressure rise at close to $x / c=0.5$, with the elevated ground exhibiting a slightly diffused version of this interaction due to the 'cushioning' effect of the boundary layer. The downstream effect of this is such that the elevated ground pressure distribution does not recover to the same extent as the other two, near identical pressure distributions, and would therefore exert a lingering influence into the wake region. The symmetry method does not quite capture the extent of the suction peak, and thus a clear connection between very small ground clearances and increasing discrepancies between the symmetry and moving ground boundary representations emerges. Interestingly, although the elevated ground plate clearly causes a large, unwanted disruption to the flow at the leading edge, the predicted ground pressure distribution in the CFD soon aligns with the symmetry and moving ground distributions. The dominant pressure gradient produced by the actual wing therefore seems to have a useful settling effect on the leading edge flow, although the influence of the elevated ground on the upstream flow remains.

Without the ability in this particular program to conduct more detailed flowfield analysis using laser measurement techniques such as LDA or PIV, CFD proved to be an essential tool in analyzing and understanding the experimental results, leading to a much improved approach to aspects of the numerical study such as mesh refinement and implementation of turbulence model and discretization. With the CFD validated in this way, a more detailed study could be conducted, investigating far more variables than would be practical in a wind tunnel program.

\section{Conclusions}

These example of supersonic and transonic ground effect aerodynamics research demonstrate the successful integration of CFD and EFD. 
For subsonic ground effect aerodynamics, a moving ground is accepted as the best method for simulation of the ground boundary conditions. However for high speed applications, this method is not feasible; alternative methods of an elevated ground plane and a 'mirror image' symmetry model were studied to determine their suitability, with the symmetry approach determined to be the most effective when experimental and numerical programs were combined in analysis.

The use of CFD facilitated better quantification of the differences between the two experimental methods, and led to an enhanced understanding of the complexity of the flow not immediately apparent from the raw data from the testing. In using CFD to initially design, then support, and finally extend the investigation to Reynolds numbers and combinations of variables not possible in the wind tunnel, the benefits of such close coordination between the two techniques become clear.

\section{References}

[1] P. Cook, M. A. McDonald, M. C. P. Firmin. Aerofoil RAE 2822 - Pressure distributions and boundary layer and wake measurements. Experimental data base for computer program assessment. AGARD Report AR 138, 1979. C266, C272

[2] A. Garbaruk, M. Shur, M. Strelets, P. Spalart. Numerical study of wind-tunnel walls effects on transonic airfoil flow. AIAA Journal, 41(6):1046-1054, 2003. C266

[3] T. Barber, E. Leonardi, and R. Archer. Causes for discrepancies in ground effect analyses. The Aeronautical Journal, 106(1066):653-657, 2002. C268 
[4] B. Fago, H. Lindner, O. Mahrenholtz. The effect of ground simulation on the flow around vehicles in wind tunnel testing. Journal of Wind Engineering and Industrial Aerodynamics, 38:47-57, 1991. C268

[5] J. Lamb. Critical velocities for rocket sled excitation for rail resonance. Johns Hopkins APL Technical Digest, 21(3):448-458. 2001. C268

[6] J. Purdon, N. Mudford, H. Kleine. Supersonic projectiles in the vicinity of solid obstacles. Proc. 27th Int. Congr. High-Speed Photography and Photonics, SPIE, Bellingham, vol. 6279, 2007. C268

[7] K. Rozhdestvensky. Wing-in-ground effect vehicles. Progress in Aerospace Sciences, 42(3):211-283, 2006. C269

[8] J. Powell, G. Maise, J. Paniagua, J. Rather, Maglev launch and the next race to space. IEEE Aerospace Conference Proceedings, Big Sky, USA, 2008. C269

[9] R. S. Raghunathana, H. -D. Kim and T. Setoguchic. Aerodynamics of high-speed railway train. Progress in Aerospace Sciences, 38:6-7, 2002. C269

[10] G. Doig. Compressible ground effect aerodynamics. PhD Thesis. University of New South Wales, Sydney Australia. 2009. C269

[11] G. Doig, T. Barber, E. Leonardi, A. Neely, H. Kleine. Methods for investigating supersonic ground effect in a blowdown wind tunnel. Shock Waves, 18(2):155-159. C269

[12] G. Doig, T. Barber, E. Leonardi, A. Neely. An investigation of flow field interactions with the ground around a supersonic land speed record vehicle using thermochromic liquid crystals and CFD. Proc. International Heat Transfer Conference, IHTC-13, Sydney, Australia, 13-18 August 2006. ISBN 1-56700-226-9, paper EXP-19, 2006. C272 
[13] D. Aeschliman, W. Oberkampf. Experimental methodology for computational fluid dynamics code validation. AIAA Journal, 36(5):733-741, 1998. C272

\section{Author addresses}

1. G. Doig, School of Mechanical \& Manufacturing Engineering, University of New South Wales, Sydney, NSW 2052, Australia.

2. T. Barber, School of Mechanical \& Manufacturing Engineering, University of New South Wales, Sydney, NSW 2052, Australia. mailto:t. barber@unsw.edu . au

3. A. Neely, School of Engineering \& Information Technology, University of New South Wales, Australian Defence Force Academy, Canberra, ACT 2600, Australia.

4. D. Myre, Aerospace Engineering Department, The United States Naval Academy, 590 Holloway Road, 11B, Annapolis, MD 21402, USA 